\title{
Desempeño profesional en relación con el síndrome de burnout de los docentes desde la percepción de los estudiantes de maestría de la escuela de postgrado de la universidad nacional de educación Enrique Guzmán y Valle
}

\section{Professional performance in relation to the teachers' burnout syndrome from the perception of the masters students of the graduate school of the national university of education Enrique Guzman y Valle}

Kriss Melody Calla Vásquez

Universidad César Vallejo, Perú

Autor para correspondencia: kcalla@ucv.edu.pe

Fecha de recepción: 01 de agosto de 2018 - Fecha de aceptación: 02 octubre de 2018

Resumen: Se realizó un estudio para obtener un diagnóstico de los elementos del Desempeño Profesional y el Síndrome de Burnout de los docentes del área de investigación, desde la percepción de los estudiantes de maestría de la Escuela de Postgrado de la Universidad Nacional de Educación Enrique Guzmán y Valle, utilizando dos instrumentos de recolección de datos, en una muestra probabilística de 268 estudiantes durante el periodo académico 2010-I. Dicho estudio estuvo basado en el análisis de la variable: Desempeño Profesional, que abarco cuatro importantes dimensiones, como son: Capacidades pedagógicas, habilidades metodológicas, responsabilidad en el desempeño de sus funciones laborales, y, relaciones interpersonales con la comunidad educativa; asimismo el análisis para la variable: Síndrome de Burnout, se dimensiono en tres importantes factores: Cansancio emocional, despersonalización y realización personal.

Palabras Claves: burnout; desempeño profesional

\begin{abstract}
A study was conducted to obtain a diagnosis of the elements of the professional performance and the syndrome of Burnout of teachers in the area of research, from the perception of the students of master's degree from the Graduate School of the National University of Education Valley, using two data collection instruments, and Enrique Guzman using two data collection instruments, in a sample probability of 268 students during the academic period 2010I. this study was based on analysis of the variable: professional performance, which included four major dimensions, such as: pedagogical capacities, methodological skills, responsibility in the performance of their job duties, and, interpersonal relationships with the educational community; also the analysis for the variable: Burnout Syndrome, dimensiono on three important factors: emotional exhaustion, depersonalization and personal accomplishment.
\end{abstract}

Key Words: burnout; professional performance 


\section{Introducción}

La investigación se desarrolló durante un año académico, en la primera etapa, se aplicó la prueba piloto a un grupo con similares características de nuestra muestra real, con la finalidad de demostrar la fiabilidad de nuestros instrumentos de medición de las variables; en la segunda etapa (fin del periodo académico 2010-I), trabajamos con la muestra completa hallado mediante la fórmula probabilística para muestras finitas, la misma que nos permitió tener la certeza que englobábamos el total de la población.

El presente trabajo de investigación tiene la siguiente estructura:

El capítulo I, comprende el marco teórico, sobre la base de cada una de las variables materia de investigación tales como: El desempeño profesional, el síndrome de burnout y la educación superior.

El capítulo II, describe el problema objeto de la investigación, su formulación, la importancia y las limitaciones en el desarrollo de la misma.

El capítulo III, propone los objetivos, describe los sistemas de hipótesis y variables, detalla el tipo y método utilizado en la investigación, explica el diseño de la investigación y describe la población y la muestra.

El capítulo IV, describe los instrumentos de recolección de datos empleados en la investigación, que permitirá bajo similares condiciones que otros investigadores puedan replicar el mismo tipo de investigación, y así acercarse a similares resultados.

Por otro lado, los instrumentos empleados en la recolección de datos, son descritos con amplitud, detallando las características que presenta cada uno de ellos, así como los análisis de validez y confiabilidad, además se muestra los resultados bajo procedimientos estadísticos, seguido de la presentación de cuadros, los mismos que han sido analizados e interpretados para dar paso a las conclusiones de la investigación.

En suma este capítulo hace referencia a qué es lo que finalmente se encontró al término de la investigación; que significan realmente los resultados obtenidos, señalando la relación existente entre los hechos observados, en este caso, el efecto de la variable independiente en la variable dependiente. Así como también se hace referencia a la discusión de los resultados. Luego de todo, se pudo dar las conclusiones y recomendaciones.

\section{Metodología}

La presente investigación, según su función última, es de tipo descriptiva, puesto que se orienta a caracterizar o establecer cómo son las relaciones existentes entre las dimensiones del desempeño profesional y el síndrome de burnout de los docentes, desde la percepción de los estudiantes de maestría, en el caso concreto establecido. De acuerdo con Best (1982), la investigación trata de informar acerca del estado actual del fenómeno educativo estudiado en calidad de problema de investigación, caracterizándolo a través de los rasgos esenciales de las 
relaciones antedichas, tales como su existencia y magnitud o intensidad, derivando a partir de todo ello, conclusiones significativas.

Asimismo según Kerlinger (2002), Sánchez \& Reyes (2006), y Hernández (2008), esta es una investigación de tipo no experimental puesto que no se introduce variable experimental alguna ni se manipula o varía arbitrariamente la variable independiente sobre la dependiente.

El método empleado en este trabajo fue:

a) Analítico-sintético: porque se estudiaron de modo empírico-teórico cada uno de los aspectos esenciales del tema estudiado, descomponiéndolos en sus elementos, llegando posteriormente a determinados niveles de integración, abstracción, caracterización y generalización;

b) Inductivo-deductivo: porque partimos de los hechos o realidades educativas concretas para definir el problema, categorizar y conceptuar; luego volvimos a los hechos con reiteración, pero cada vez mejor equipados teóricamente, ya sea en función de recolección de datos, estudio interpretativo, verificatorio, valorativo, descriptivo, de contraste y otros;

c) Analítico-descriptivo: porque cada elemento producto del análisis (variables e indicadores, por ejemplo), se sometieron a un proceso de descripción con propósitos de definición, conceptuación y caracterización.

El diseño empleado en esta investigación fue el descriptivo-correlacional, de corte transeccional o transversal, puesto que se registró información acerca de las variables bajo estudio (desempeño profesional y el síndrome de burnout de los docentes) con el objetivo de determinar el grado de correlación que existe entre las dos variables de interés en una misma muestra de sujetos, cuyo diagrama es el siguiente:

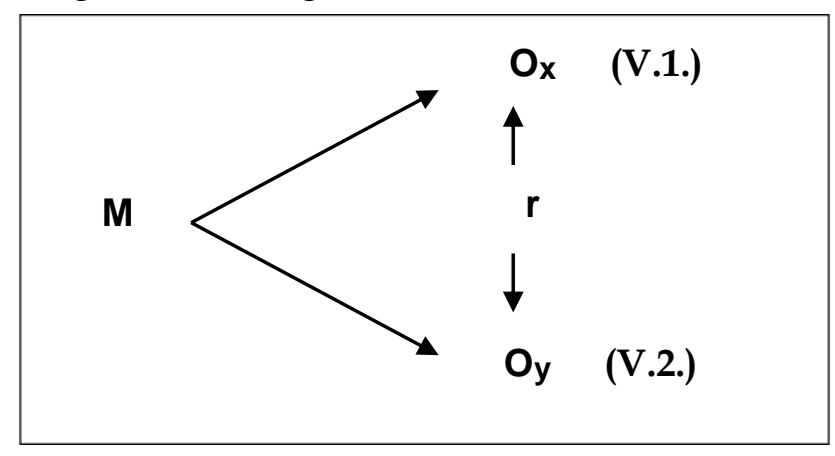

Diagrama $\mathbf{N}^{\circ} \mathbf{1}$

Diseño de Investigación Descriptivo - Correlacional

\section{En donde:}

$\begin{array}{lll}\mathbf{M} & = & \text { Muestra de Investigación. } \\ \mathbf{O}_{\mathbf{x}} & = & \text { Variable 1. (Desempeño Profesional) } \\ \mathbf{O}_{\mathbf{y}} & = & \text { Variable } 2 . \text { (Síndrome de Burnout) } \\ \mathbf{r} & = & \text { Relación entre variables. }\end{array}$


Para los fines de la presente investigación la población estuvo conformado por $\mathbf{8 8 2}$ estudiantes de maestría de la Escuela de Postgrado de la Universidad Nacional de Educación Enrique Guzmán y Valle, matriculados en las asignaturas del área de investigación, distribuidos en 10 menciones, 4 promociones y 29 secciones

Para el caso de los estudiantes se tomó una muestra probabilística al 95\% de seguridad estadística, cuya fórmula para muestras finitas es la siguiente:

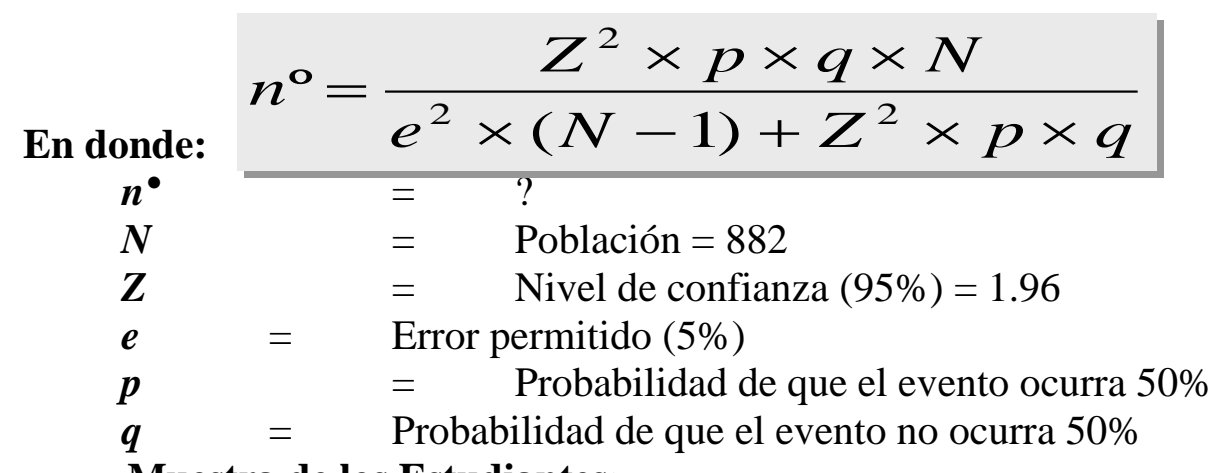

$$
\begin{aligned}
& \text { Muestra de los Estudiantes: } \\
& n^{\mathbf{o}}=\frac{1,96^{2} \times 0,5 \times 0,5 \times 882}{0,05^{2} \times n^{\mathbf{o}}=268,96^{2} \times 0,5 \times 0,5}
\end{aligned}
$$

\section{Resultados}

\section{Cuadro $\mathbf{N}^{0} 01$}

Consolidado general sobre el cuestionario de opinión de los estudiantes, respecto al desempeño profesional de los docentes del área de investigación, desde la percepción de los estudiantes de maestría de la Escuela de Postgrado de la Universidad Nacional de Educación

\begin{tabular}{|c|c|c|c|c|c|}
\hline ÍTEMS & Nunca & $\begin{array}{r}\text { Casi } \\
\text { Nunca }\end{array}$ & A Veces & $\begin{array}{c}\text { Casi } \\
\text { Siempre }\end{array}$ & Siempre \\
\hline & $\%$ & $\%$ & $\%$ & $\%$ & $\%$ \\
\hline $\begin{array}{l}\text { 1. Posee capacidad para hacer su materia } \\
\text { entretenida e interesante. }\end{array}$ & 3.73 & 20.52 & 44.40 & 28.36 & 2.99 \\
\hline $\begin{array}{l}\text { 2. Demuestra capacidad para planificar } \\
\text { adecuadamente el proceso docente - educativo. }\end{array}$ & 0.37 & 16.79 & 67.54 & 13.81 & 1.49 \\
\hline $\begin{array}{l}\text { 3. Tiene la capacidad de contribuir a un adecuado } \\
\text { clima de trabajo en el aula. }\end{array}$ & 1.87 & 6.72 & 43.28 & 40.67 & 7.46 \\
\hline $\begin{array}{l}\text { 4. Posee capacidad para identificar, comprender las } \\
\text { situaciones que ocurre en el aula y ajustar su } \\
\text { intervención pedagógica. }\end{array}$ & 4.85 & 30.60 & 42.91 & 19.40 & 2.24 \\
\hline $\begin{array}{l}\text { 5. Contribuye en la formación de valores } \\
\text { nacionales, universales y al desarrollo de } \\
\text { capacidades valorativas. }\end{array}$ & 1.49 & 21.64 & 47.76 & 26.87 & 2.24 \\
\hline $\begin{array}{l}\text { 6. Tiene capacidad para crear un ambiente } \\
\text { favorable para que el estudiante conozca sus }\end{array}$ & 1.49 & 11.94 & 53.73 & 32.09 & 0.75 \\
\hline
\end{tabular}
Enrique Guzmán y Valle, durante el periodo académico 2010-I. 
derechos y responsabilidades y aprenda a ejercerlos.

7. Posee capacidad para desarrollar un proceso de $\quad \begin{array}{lllll}0.75 & 7.84 & 53.73 & 36.94 & 0.75\end{array}$ reflexión autocrítica permanente sobre su práctica educativa.

8. Domina los contenidos que imparte con claridad y amplitud.

9. Posee buena calidad de comunicación verbal y no verbal.

10. Utiliza gran variedad de prácticas educativas.

11. Posee un alto grado de conocimientos y tratamientos de las características psicológicas individuales de los estudiantes.

12. Tiene un alto grado de información sobre la marcha del aprendizaje de sus estudiantes.

13. Demuestra calidad de representación sobre el cargo social de la Escuela de Postgrado.

14. Transmite la efectividad de su capacitación y autopreparación.

15. Posee vocación pedagógica.

16. Tiene Autoestima.

4.85

16.04

46.27

29.85

2.99

0.37

15.67

$66.42 \quad 15.67$

1.87

$\begin{array}{lllll}0.75 & 6.34 & 37.31 & 44.40 & 11.19\end{array}$

$\begin{array}{lllll}3.73 & 31.72 & 38.43 & 23.88 & 2.24\end{array}$

$\begin{array}{lllll}0.75 & 21.64 & 47.76 & 27.24 & 2.61\end{array}$

$\begin{array}{lllll}0.37 & 8.58 & 56.34 & 32.84 & 1.87\end{array}$

$\begin{array}{lllll}0.00 & 7.09 & 51.12 & 40.30 & 1.49\end{array}$

$\begin{array}{lllll}5.60 & 15.30 & 45.52 & 28.36 & 5.22\end{array}$

$\begin{array}{lllll}0.37 & 15.30 & 55.22 & 22.76 & 6.34\end{array}$

17. Demuestra capacidad para actuar con justicia y realismo.

$\begin{array}{lllll}1.87 & 8.58 & 44.78 & 38.81 & 5.97\end{array}$

18. Presenta buen nivel de satisfacción con la labor que realiza.

19. Asiste puntualmente a la Escuela y a sus clases.

4.10

13.81

50.00

25.75

6.34

0.00

14.18

58.96

22.76

4.10

20. Participa en las sesiones metodológicas o en

$1.49 \quad 7.46$

47.39

38.06

5.60 jornadas de reflexión entre los docentes.

21. Cumple todas las normativas que le establecen.

6.34

21.27

38.81

30.97

2.61

22. Demuestra un alto nivel profesional alcanzado.

23. Tiene implicancia personal en la toma de decisiones de la Escuela.

24. Demuestra autonomía profesional relativa alcanzada para desarrollar su tarea en la Escuela.

25. Se preocupa y comprende los problemas de sus estudiantes.

26. Tiene altos niveles de expectativas respecto al desarrollo de sus estudiantes.

27. Demuestra flexibilidad para aceptar la diversidad de opinión y sentimientos de los estudiantes y respeto real por sus diferencias de género, raza, y situación socioeconómica.

28. Tiene estudiantes que han alcanzado un alto rendimiento académico en la asignatura que imparte.

$\begin{array}{lllll}4.48 & 14.93 & 57.84 & 21.64 & 1.12\end{array}$

$\begin{array}{lllll}0.75 & 8.96 & 48.88 & 37.31 & 4.10\end{array}$

$\begin{array}{lllll}3.36 & 15.67 & 48.88 & 28.36 & 3.73\end{array}$

$\begin{array}{lllll}3.36 & 10.45 & 41.04 & 34.70 & 10.45\end{array}$

$\begin{array}{lllll}3.73 & 17.54 & 51.49 & 21.64 & 5.60\end{array}$

$\begin{array}{lllll}0.75 & 14.18 & 53.73 & 25.75 & 5.60\end{array}$

$\begin{array}{lllll}3.36 & 12.69 & 42.54 & 33.96 & 7.46\end{array}$

$\begin{array}{lllll}4.85 & 22.76 & 42.16 & 27.61 & 2.61\end{array}$

29. Posee un alto grado de orientación valorativa positiva, alcanzado hacia las cualidades deseables de la personalidad de acuerdo con el modelo de hombre que se pretende formar. 


\begin{tabular}{lccccc}
\hline $\begin{array}{l}\text { 30. Tiene estudiantes que poseen sentimientos de } \\
\text { amor a la patria, a la naturaleza, al estudio y al }\end{array}$ & 2.99 & 22.01 & 48.88 & 24.63 & 1.49 \\
género humano. & & & & & \\
& $\mathbf{7 2 . 3 0}$ & $\mathbf{4 6 5 . 0 0}$ & $\mathbf{1 4 6 8 . 8 0}$ & $\mathbf{8 7 3 . 3 0}$ & $\mathbf{1 2 0 . 6 0}$ \\
Porcentaje Promedio & 2.41 & 15.50 & 48.96 & 29.11 & 4.02 \\
\hline
\end{tabular}

Elaborado por Kriss Melody Calla Vásquez.

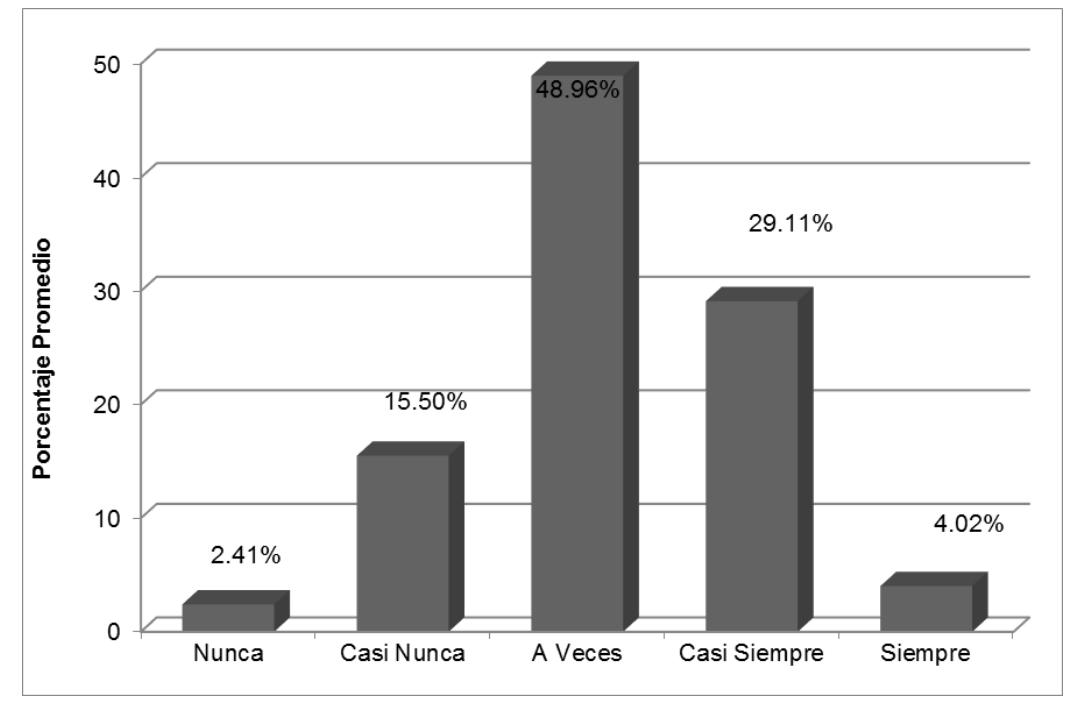

Gráfico Del Cuadro $\mathrm{N}^{\circ} 01$

\section{Resumen general del cuestionario sobre el desempeño profesional}

\begin{tabular}{|c|c|c|c|c|c|c|}
\hline Variable & & Nunca & $\begin{array}{r}\text { Casi } \\
\text { Nunca }\end{array}$ & $\begin{array}{c}\text { A } \\
\text { Veces }\end{array}$ & $\begin{array}{c}\text { Casi } \\
\text { Siempre }\end{array}$ & Siempre \\
\hline \multirow{6}{*}{ 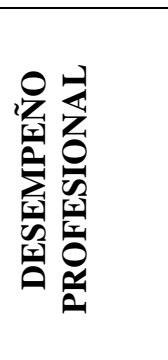 } & Capacidades pedagógicas & 2.08 & 16.58 & 50.48 & 28.30 & 2.56 \\
\hline & Habilidades metodológicas & 1.55 & 15.30 & 49.09 & 30.60 & 3.46 \\
\hline & $\begin{array}{l}\text { Responsabilidad en el } \\
\text { desempeño de sus funciones }\end{array}$ & 2.84 & 13.54 & 49.63 & 29.48 & 4.51 \\
\hline & laborales & & & & & \\
\hline & $\begin{array}{l}\text { Relaciones interpersonales } \\
\text { con la }\end{array}$ & 3.17 & 16.60 & 46.64 & 28.05 & 5.53 \\
\hline & Comunidad educativa & 241 & 1550 & 1806 & 2011 & 09 \\
\hline
\end{tabular}

Fuente: Anexo $\mathrm{N}^{\circ} 06$

Elaborado por Kriss Melody Calla Vásquez.

\section{Explicación}

Objetivo: El objetivo de los resultados mostrados en el gráfico $\mathrm{N}^{\mathrm{o}} 07$, es determinar el nivel de la variable: Desempeño Profesional de los docentes del área de investigación, desde la percepción de los estudiantes de maestría de la Escuela de Postgrado de la Universidad Nacional de Educación Enrique Guzmán y Valle, durante el periodo académico 2010-I.

\section{Interpretación:}


Teniendo en cuenta la totalidad de los ítems, se puede llegar a una apreciación general sobre el desempeño. Según el cuadro, se observa que el $33.13 \%$ de los estudiantes está de acuerdo con el desempeño profesional de los docentes del área de investigación de la Escuela de Postgrado de la Universidad Nacional de Educación Enrique Guzmán y Valle, donde el 4.02\% de los mismos, considera que los docentes "Siempre" demuestran poseer un alto desempeño, en tanto que para el 29.11\% "Casi Siempre" lo demuestran. Por otro lado, una gran mayoría equivalente al $48.96 \%$ de los estudiantes opina que "A veces" el docente demuestra poseer un buen nivel de desempeño profesional, mientras que una minoría equivalente al $17.91 \%$ descalifica su desempeño. En síntesis, se llega a la conclusión de que los docentes del área de investigación de la Escuela de Postgrado de la Universidad Nacional de Educación Enrique Guzmán y Valle, $\underline{A}$ $\underline{\text { veces, }}$ demuestran poseer un alto desempeño profesional.

\section{Conclusión}

Por lo tanto, se concluye diciendo que de acuerdo a la percepción de los estudiantes, los docentes del área de investigación de la Escuela de Postgrado de la Universidad Nacional de Educación Enrique Guzmán y Valle, durante el periodo académico 2010-I, A veces demuestran un buen nivel de desempeño profesional.

\section{Consolidado general del cuestionario sobre: El Síndrome De Burnout en los docentes}

\section{Cuadro $\mathrm{N}^{\circ} 02$}

Consolidado general sobre el cuestionario de opinión de los estudiantes, respecto a la prevalencia del síndrome de burnout de los docentes del área de investigación, desde la percepción de los estudiantes de maestría de la Escuela de Postgrado de la Universidad Nacional de Educación Enrique Guzmán y Valle, durante el periodo académico 2010-I.

\begin{tabular}{|c|c|c|c|c|c|}
\hline ÍTEMS & Nunca & $\begin{array}{r}\text { Casi } \\
\text { Nunca }\end{array}$ & A Veces & $\begin{array}{c}\text { Casi } \\
\text { Siempre }\end{array}$ & Siempre \\
\hline & $\%$ & $\%$ & $\%$ & $\%$ & $\%$ \\
\hline $\begin{array}{l}\text { 1. Sientes emocionalmente agotado al } \\
\text { docente en su trabajo. }\end{array}$ & 3.73 & 16.42 & 54.48 & 21.64 & 3.73 \\
\hline $\begin{array}{l}\text { 2. Sientes al docente cansado al final de su } \\
\text { jornada de trabajo. }\end{array}$ & 2.24 & 11.19 & 51.49 & 28.36 & 6.72 \\
\hline $\begin{array}{l}\text { 3. Sientes al docente fatigado cuando inicia } \\
\text { su trabajo de clases. }\end{array}$ & 2.99 & 12.69 & 46.27 & 31.72 & 6.34 \\
\hline $\begin{array}{l}\text { 4. Percibes que trabajar todo el día con } \\
\text { mucha gente es un esfuerzo para el } \\
\text { docente. }\end{array}$ & 2.61 & 14.93 & 55.22 & 22.76 & 4.48 \\
\hline $\begin{array}{l}\text { 5. Sientes agotado "quemado" al docente en } \\
\text { su trabajo. }\end{array}$ & 1.12 & 12.31 & 56.34 & 26.12 & 4.10 \\
\hline 6. Sientes frustrado al docente en su trabajo. & 4.10 & 12.69 & 45.52 & 33.21 & 4.48 \\
\hline $\begin{array}{l}\text { 7. Sientes que el docente está trabajando } \\
\text { demasiado. }\end{array}$ & 6.34 & 18.28 & 46.64 & 26.87 & 1.87 \\
\hline
\end{tabular}


8. Percibes que trabajar directamente con

\begin{tabular}{|c|c|c|c|c|}
\hline 2.61 & 11.19 & 55.97 & 29.10 & 1.12 \\
\hline 1.49 & 8.96 & 49.63 & 36.57 & 3.36 \\
\hline 1.87 & 11.94 & 45.90 & 33.96 & 6.34 \\
\hline 2.99 & 12.31 & 56.34 & 23.51 & 4.85 \\
\hline 1.49 & 11.94 & 55.22 & 27.24 & 4.10 \\
\hline 0.75 & 12.69 & 46.64 & 33.96 & 5.97 \\
\hline 1.12 & 14.18 & 54.48 & 27.99 & 2.24 \\
\hline 3.73 & 35.07 & 36.19 & 23.13 & 1.87 \\
\hline 0.75 & 45.90 & 38.06 & 14.55 & 0.75 \\
\hline 2.61 & 37.31 & 34.70 & 22.39 & 2.99 \\
\hline 4.48 & 36.19 & 38.06 & 19.40 & 1.87 \\
\hline 1.49 & 39.55 & 37.69 & 20.15 & 1.12 \\
\hline 1.87 & 34.33 & 36.94 & 24.25 & 2.61 \\
\hline 4.48 & 37.69 & 38.43 & 17.54 & 1.87 \\
\hline 2.61 & 45.90 & 33.96 & 16.79 & 0.75 \\
\hline 54.34 & 474.98 & 1024.98 & 568.92 & 76.56 \\
\hline 2.47 & 21.59 & 46.59 & 25.86 & 3.48 \\
\hline
\end{tabular}

Porcentaje Promedio

Elaborado por Kriss Melody Calla Vásquez. 


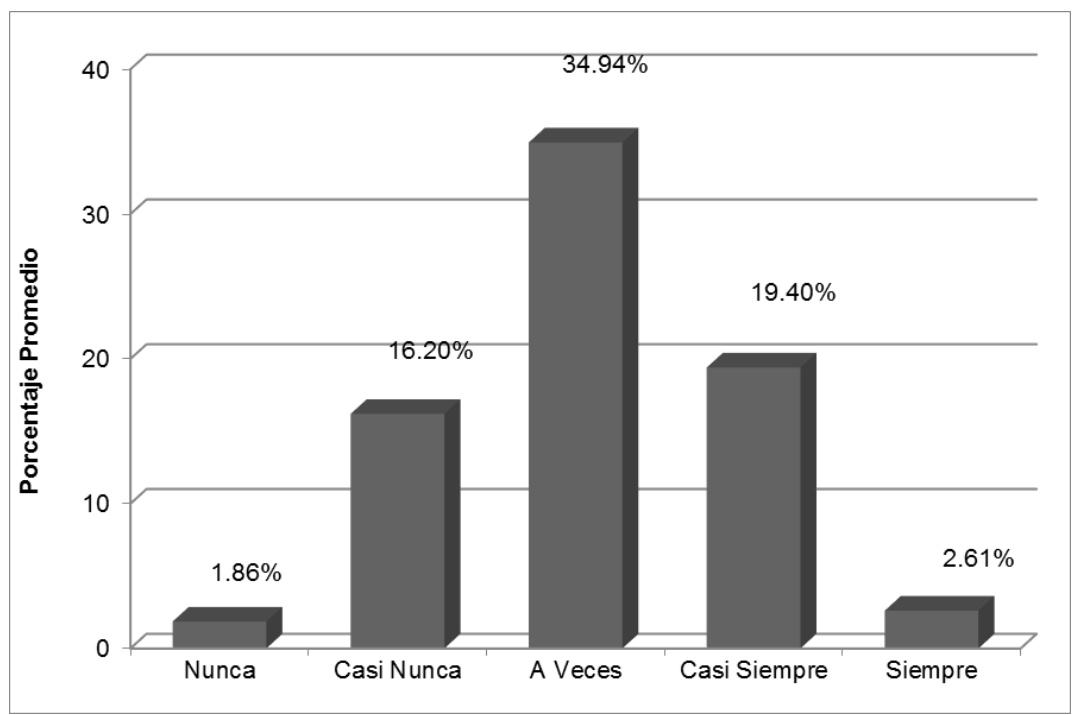

Gráfico Del Cuadro Nº 02

Resumen general del cuestionario sobre: EI Síndrome De Burnout de los docentes

\begin{tabular}{|c|c|c|c|c|c|c|}
\hline Variable & & Nunca & Casi Nunca & A Veces & Casi Siempre & Siempre \\
\hline \multirow{4}{*}{ 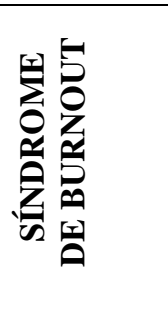 } & Cansancio emocional & 3.03 & 13.18 & 51.29 & 28.48 & 4.02 \\
\hline & Despersonalización & 1.64 & 12.61 & 51.72 & 29.33 & 4.70 \\
\hline & Realización personal & 2.75 & 38.99 & 36.75 & 19.78 & 1.73 \\
\hline & & 2.47 & 21.59 & 46.59 & 25.86 & 3.48 \\
\hline
\end{tabular}

Fuente: Anexo $\mathrm{N}^{\circ} 06$

Elaborado por Kriss Melody Calla Vásquez.

\section{Explicación}

\section{Objetivo:}

El objetivo de los resultados mostrados en el gráfico del cuadro $\mathrm{N}^{\mathrm{0}} 11$, es determinar el nivel de la variable: Síndrome de Burnout de los docentes del área de investigación, desde la percepción de los estudiantes de maestría de la Escuela de Postgrado de la Universidad Nacional de Educación Enrique Guzmán y Valle, durante el periodo académico 2010-I.

\section{Interpretación:}

Teniendo en cuenta la totalidad de los ítems, se puede llegar a una apreciación general sobre la presencia del síndrome de burnout en los docentes del área de investigación, desde la percepción de los estudiantes. Según el cuadro, se observa que el $29.34 \%$ de los encuestados, percibe la presencia del síndrome de burnout en los docentes del área de investigación de la Escuela de Postgrado de la Universidad Nacional de Educación Enrique Guzmán y Valle, donde el $3.48 \%$ de los mismos considera que "Siempre" lo percibe, en tanto que el $25.86 \%$ "Casi 
Siempre" lo percibe. Por otro lado, una gran mayoría equivalente al $46.59 \%$ de los estudiantes opina que "A veces" percibe la presencia del síndrome de burnout en los docentes, mientras que una minoría equivalente al $24.06 \%$ no percibe dicha presencia. En síntesis, se llega a la conclusión de que los docentes del área de investigación de la Escuela de Postgrado de la Universidad Nacional de Educación Enrique Guzmán y Valle, A veces, presentan el síndrome de burnout.

\section{Conclusión}

Por lo tanto se concluye diciendo que de acuerdo a la percepción de los estudiantes, los docentes del área de investigación de la Escuela de Postgrado de la Universidad Nacional de Educación Enrique Guzmán y Valle, durante el periodo académico 2010-I, A veces, presentan el síndrome de burnout.

\section{Discusión}

La presente investigación a través de los resultados obtenidos, ha encontrado que el desempeño profesional guarda relación significativa con el síndrome de burnout de los docentes del área de investigación, desde la percepción de los estudiantes de maestría de la Escuela de Postgrado de la Universidad Nacional de Educación Enrique Guzmán y Valle, durante el periodo académico 2010-I

No obstante, los resultados en cuanto al nivel descriptivo, muestra que en lo referente al desempeño profesional, los resultados hallados son congruentes o compatibles con otros a nivel de evaluaciones, de acuerdo con Del Castillo (2008), quien en su estudio sobre el desempeño docente, encuentra que el nivel bajo de desempeño equivale al $27.93 \%$, el nivel medio con tendencia declinante $52.41 \%$ y nivel alto $19.66 \%$, esto es congruente con nuestros resultados obtenidos, puesto que los estudiantes de maestría de la Escuela de Postgrado de la Universidad Nacional de Educación Enrique Guzmán y Valle, de acuerdo a sus percepciones, ubican a sus maestros en los siguientes niveles de desempeño (Nunca y Casi Nunca) equivalente al 17.91\%, asimismo un predominante nivel promedio de desempeño (A Veces) equivalente al 48.96\%, y en el nivel de alto desempeño (Siempre y Casi Siempre) equivalente al 33.13\%, este resultado es explicable por que la psicología de los estudiantes está cada vez unida a sus emociones, susceptible a factores no directamente relacionados con la calidad de la tarea docente (criterios de empatía) considerando además que los estudiantes tienen escasa o ninguna experiencia para evaluar los aspectos cualitativos de la enseñanza, muchas veces podrían haber utilizado este momento como espacio para dirimir situaciones externas al proceso educativo.

Respecto a la presencia del síndrome de burnout en los docentes, desde la percepción de los estudiantes, el resultado más alto estuvo ubicado en el nivel intermedio de $\underline{\mathrm{A} \text { Veces }}$ equivalentes al $46.59 \%$, asimismo, otra mayoría de los estudiantes de maestría equivalentes al 29.34\% percibe que Casi Siempre y Siempre los docentes, presentan el síndrome de burnout, por otro lado un menor grupo de la muestra de estudiantes encuestados equivalentes al $24.06 \%$ percibe que Casi Nunca y Nunca, los docentes del área de investigación de la Escuela de Postgrado de la Universidad 
Nacional de Educación Enrique Guzmán y Valle, presentan el síndrome de burnout. Estos resultados son congruentes de acuerdo a lo establecido por Maslach \& Jackson (1981), quienes consideran que los límites normales de burnout (presencia del síndrome de burnout) son equivalentes al 33\% (Casi Nunca), definiendo que: “...Uno de cada tres profesionales se encuentra dentro de los límites normales de burnout (33\%), otro se encuentra quemado (34\%) y la tercera se puede considerar muy quemado (33\%), con dos o tres factores alterados...", por lo que concluimos que existe en los docentes del área de investigación de la Escuela de Postgrado la presencia y prevalencia del síndrome de burnout.

Estos resultados obtenidos son congruentes con la teoría del síndrome de burnout estudiado por Maslach \& Jackson (1981), quienes lo definen como: "un síndrome caracterizado por la existencia de factores como: cansancio emocional, despersonalización y baja realización personal que puede presentarse en profesionales que desempeñan cualquier actividad laboral que tenga como objetivo la atención a otras personas". Asimismo, de acuerdo con Ramírez (1995), se considera como prevalencia de burnout el tener uno o más factores alterados en un profesional, basándonos en otros estudios que han utilizado este criterio. Por otro lado para Gil-Monte, (2000), existen además unos criterios normativos para muestras de profesionales de la salud. 


\section{Conclusiones}

Concluido el trabajo de investigación podemos establecer las siguientes conclusiones:

El desarrollo de la presente tesis, en base a la presentación de los datos y los análisis estadísticos, nos permite alcanzar las siguientes conclusiones:

La investigación de acuerdo a los resultados estadísticos inferenciales, señalan que se acepta la Hipótesis General concluyéndose que: Existe relación significativa entre el Desempeño Profesional y el Síndrome de Burnout de los docentes del área de investigación, desde la percepción de los estudiantes de maestría de la Escuela de Postgrado de la Universidad Nacional de Educación Enrique Guzmán y Valle, durante el periodo académico 2010-I.

Los datos obtenidos de las encuestas y los resultados inferenciales, señalan que se acepta la Hipótesis Especifica $N^{\circ} 1$, concluyéndose que: Existe relación significativa entre el Desempeño Profesional y el Cansancio Emocional de los docentes del área de investigación, desde la percepción de los estudiantes de maestría de la Escuela de Postgrado de la Universidad Nacional de Educación Enrique Guzmán y Valle, durante el periodo académico 2010-I.

Los datos obtenidos de las encuestas y los resultados inferenciales, señalan que se acepta la Hipótesis Especifica $\mathrm{N}^{\circ}$ 2, concluyéndose que: Existe relación significativa entre el Desempeño Profesional y la Despersonalización de los docentes del área de investigación, desde la percepción de los estudiantes de maestría de la Escuela de Postgrado de la Universidad Nacional de Educación Enrique Guzmán y Valle, durante el periodo académico 2010-I.

Los datos obtenidos de las encuestas y los resultados inferenciales, señalan que se acepta la Hipótesis Especifica $N^{\circ} 3$, concluyéndose que: Existe relación significativa entre el Desempeño Profesional y la Realización Personal de los docentes del área de investigación, desde la percepción de los estudiantes de maestría de la Escuela de Postgrado de la Universidad Nacional de Educación Enrique Guzmán y Valle, durante el periodo académico 2010-I.

\section{Bibliografía}

Arévalo, G. \& Otros (2000). La investigación científica en educación. Lima: Ediciones Interconed.

Barría, M. (2002). Síndrome de Burnout en asistentes sociales del Servicio Nacional de Menores de la Región Metropolitana de Chile. Chile.

Bastos, E. (1998). Educación en Valores. Lima: Editorial CEPROC.

Bretel, L. (2002). Propuesta para el diseño de un sistema de evaluación del desempeño docente en el marco de una redefinición de la carrera pública magisterial. Santiago de Chile: Ediciones MINEDUC.

Burke, R.J. \& Greenglass, E.R. (1995). A longitudinal study of psychological burnout in teachers. Human Relations, 48, 187-202. 
Caramés, R. (2001). Causas del core of Burnout del Profesorado Universitario. Investigación no publicada, Universidad de Coruña, La Coruña, España.

Cherniss, C. (1980). Professional Burnout human service organizations. NY Praeger.

Del Castillo, V. (2008). Calidad de los componentes del desempeño docente y niveles de logro académico de los alumnos en colegios estatales de Lima Metropolitana. Tesis de Doctorado no publicada, Universidad Nacional de Educación Enrique Guzmán y Valle, Lima, Perú.

Estraño, A. (1980). Predicting Venezuelan student teacher success from academic and demographic variables available at the end of the basic cicle year. Tesis Doctoral no Publicada, The Florida State University, Tallahassee, Florida.

Fernández, M. (2008). Desempeño docente y su relación con orientación a la meta, estrategias de aprendizaje y autoeficacia: un estudio con maestros de primaria de Lima, Perú. Investigación no Publicada, Universidad de San Martín de Porres, Lima, Perú.

Fernández, M. (2008). Burnout, autoeficacia y estrés en maestros peruanos: tres estudios fácticos. Investigación no Publicada, Universidad de San Martín de Porres, Lima, Perú.

Fischer, H.J. (1983). A psicoanalytic view of burnout. En B. A. Farber (ed.), Stress and burnout in the human services professions (1985, $2^{\mathrm{a}}$ ed., pp. 40-45). New York: Pergamon Press.

Freudenberger, H.J. (1974). Staff burnout. Journal of Social Issues, 30, 159-165.

Gallardo, M. \& Maldonado, L. (2003). Estrés en docentes Universitarios. Investigación no publicada, Universidad del Zulia, Zulia, Venezuela.

Garcés de Los Fayos, Teruel \& García Montalvo (1994). Estudio comparativo de dos medidas de burnout es en personal sanitario. Anales de Psiquiatría, 10 (5), 180-184. Murcia.

García, A. (2009). Manejo del estrés laboral en profesionales, estudiantes de Postgrado de la Universidad Nacional de Educación Enrique Guzmán y Valle. Tesis de Doctorado no publicada, Universidad Nacional de Educación Enrique Guzmán y Valle, Lima, Perú.

Gil-Monte, P. \& Peiró, J.M. (1997). Desgaste psíquico en el trabajo: El síndrome de quemarse. Madrid: Síntesis.

Guerrero, E. (1996). Una investigación con docentes universitarios sobre el afrontamiento del estrés laboral y el síndrome del quemado. Investigación no publicada, Universidad de Extremadura (UEX), Extremadura, España.

Guerrero, E. (2003). Análisis pormenorizado de los grados de Burnout y técnicas de afrontamiento del estrés docente en el profesorado universitario. Investigación no publicada, Universidad de Extremadura (UEX), Extremadura, España. 
Hamachek, D. \& Otros (1970). El Character and Skills of the effective professor. Journal of Higher Education. EE.UU. Editorial Englewood Cliffs, N.J., Prentice-Hall.

Hernández, R., Fernández, C. \& Baptista, P. (2008). Metodología de la investigación (5ºd.). México: Ediciones McGraw Hill.

Hernández, S. (1960) Pedagogía Natural. Ciudad de México: Ediciones UTEHA.

Herrán, A. (2004). El Docente de baja autoestima implicaciones didácticas. Ciudad de México: Indivisa Boletín de estudios de Investigación.

Jhonson, D. (1990). Psicología Social de la Educación. Buenos Aires: Ediciones Kapelusz.

Matta, H. (2006). Clima organizacional y su relación con la prevalencia de burnout en docentes de las Facultades de Pedagogía y Cultura Física y Ciencias Administrativas y Turismo de la Universidad Nacional de Educación Enrique Guzmán y Valle. Tesis de Maestría no publicada, Universidad Nacional de Educación Enrique Guzmán y Valle, Lima, Perú. 